Cahiers de recherches médiévales

Journal of medieval studies

17 | 2009

Le poison et ses usages au Moyen Âge

\title{
Être bibliothécaire à Saint-Victor
}

Dominique Varry

\section{OpenEdition}

Journals

Édition électronique

URL : https://journals.openedition.org/crm/11527

DOI : $10.4000 / \mathrm{crm} .11527$

ISSN : 1955-2424

Éditeur

Honoré Champion

Édition imprimée

Date de publication : 15 juin 2009

Pagination : 257-270

ISSN : 1272-9752

Référence électronique

Dominique Varry, «Être bibliothécaire à Saint-Victor », Cahiers de recherches médiévales [En ligne], 17

2009, mis en ligne le 15 juin 2012, consulté le 15 décembre 2022. URL : http://

journals.openedition.org/crm/11527 ; DOI : https://doi.org/10.4000/crm.11527 


\title{
RM
}

\section{Être bibliothécaire à Saint-Victor}

\begin{abstract}
Who were the librariansof Saint-Victor in the $17^{\text {th }}$ and $18^{\text {th }}$ centuries? What were their daily practices? How did they conceive librarianship? This paper aims to answer such questions through the all-too-rare testimonies that have come down to us.

Résumé : Qui furent les bibliothécaires de Saint-Victor aux XVII et XVIII siècles? Quelles furent leurs pratiques quotidienne et leurs conceptions de la bibliothéconomie de leur temps? C'est à ces questions qu'essaie de répondre cette étude menée à partir de trop rares témoignages parvenus jusqu'à nous.
\end{abstract}

Depuis que nous avons été amené à diriger un des volumes de l'Histoire des bibliothèques françaises, notre intérêt personnel pour l'histoire du «métier» de bibliothécaire, et de ceux qui l'ont exercé, n'a fait que croître. Nous nous sommes déjà penché sur cette question en travaillant sur la période de la Révolution française. Ce fut notre première rencontre avec un bibliothécaire de Saint-Victor, François-Valentin Mulot. C'est une interrogation que nous avons reprise en organisant à l'Enssib, en 2003, un colloque sur l'histoire des bibliothécaires'. Les questions qui nous taraudent plus particulièrement sont: premièrement celle de l'élaboration d'un savoir professionnel; deuxièmement celle de la transmission de ce savoir, de génération en génération, mais aussi à des époques charnières comme celle du passage de la République des lettres au $\mathrm{XIX}^{\mathrm{e}}$ siècle. C'est ce qui nous a conduit à accepter de nous interroger sur ce en quoi consistait le fait d'être bibliothécaire à Saint-Victor, tout en étant bien conscient de la difficulté de la commande. Après avoir essayé de brosser un portrait de groupe de ces religieux bibliothécaires, nous nous interrogerons sur leur gestion quotidienne de la bibliothèque, puis essaierons de comprendre comment ces pratiques s'intégraient ou non dans le savoir bibliothéconomique de leur temps.

\section{Qui furent les bibliothécaires de Saint-Victor à l'époque moderne?}

$\mathrm{Au} \mathrm{XVII}{ }^{\mathrm{e}}$ siècle, les sept bibliothécaires qui se sont succédés ont eu une durée moyenne d'activité de treize ans. Leur liste s'établit comme suit: Jean Picard (décédé en 1615), Étienne Reynard (1615-1636), Jean de Toulouse (1636-1639), Philippe Gourreau de la Proustière (1639-1641), Jacques Bouet de la Noue (assistant en 1641, bibliothécaire 1648-ca 1651, puis à nouveau à partir de 1684), Eustache de Blémur (1651-1664), Charles Le Tonnelier (1664-1684). Certains ont exercé la charge durant peu d'années: Jean de Toulouse pendant trois ans (1636-1639), Philippe Gourreau de la Proustière pendant une période à peu près identique (1639-

${ }^{1}$ F. Barbier et D. Varry, Histoire des bibliothécaires, actes du colloque organisé à l'enssib du 27 au 29 novembre 2003, consultable en ligne sur http://www.enssib.fr/bibliothequenumerique/document-1337

Cahiers de Recherches Médiévales, 17, 2009 
1641). D'autres demeurèrent plus longtemps en poste: Étienne Reynard, prédécesseur de Jean de Toulouse, pendant vingt et un ans et jusqu'à son trépas (1615-1636). Jacques Bouet de la Noue eut deux mandats: le premier, peut-être comme assistant, de 1641 à 1648 puis comme titulaire jusqu'en 1651 environ, le second à partir de 1684 . Au XVIII ${ }^{\mathrm{e}}$ siècle, la liste est plus longue, et curieusement, les dates des fonctions sont plus mal connues. Elle compte dix-sept noms : Paul Vion d'Herouval (décédé en février 1719), l'abbé Noiret, l'abbé Lebrun, l'abbé Luce, l'abbé Bourdonne, l'abbé Contet (en charge en 1743). Son successeur fut Camille Charles Pélissier, mort en 1759 à l'âge de 45 ans dont vingt de religion, qui était licencié in utroque jure. Martin Lagrenée, qui vint ensuite, exerça jusqu'à sa mort en octobre 1762. Il était alors sexagénaire, et bachelier en théologie. Léonard Charles Brunet, qui mourut âgé de 45 ans en 1765, était docteur en théologie. Claude Cerveau, son successeur, eut un mandat très court, puisqu'il mourut en janvier 1767. Il fut aussi maître des novices et sous-prieur. Pour sa part, Armand Septier, qui reprit les fonctions à partir de 1767, pour peu de temps, mourut en 1824. Il fut, après la Révolution, l'organisateur de la bibliothèque municipale d'Orléans. Vint ensuite Nicolas Lallemant, chanoine de Saint-Cloud, qui mourut en 1771 à 65 ans. Le bibliothécaire suivant fut le célèbre abbé Mulot, qui disparut en 1804. Il devint député à la Législative... et se maria. Vinrent ensuite les abbés Joseph André Guyot, Laurent (mort en 1805), et Bruelle (mort en 1791). Le dernier titulaire, Jean Charles Marie Bernard, n'eut qu'un mandat de quelques mois, puisqu'il fut massacré au séminaire de Saint-Firmin le 3 septembre 1792. Comparativement au XVII ${ }^{\mathrm{e}}$ siècle, le temps d'exercice moyen fut plus court encore: un peu moins de six ans, mais là aussi avec de grandes variations. Une suite de décès, dans les années 1760, raccourcit ce temps. On peut simplement observer que la durée moyenne d'exercice de la charge aurait alors été bien courte pour mener une politique documentaire suivie.

\section{D'autres charges de responsabilité}

Les fonctions de bibliothécaire à Saint-Victor ont été, très anciennement, couplées à d'autres exercées simultanément. Selon Franklin² :

le chantre de l'abbaye semble avoir longtemps joint à ces fonctions celles de bibliothécaire, et c'est à ce titre seulement que nous pouvons mentionner, comme successeurs de Grandrue, Jacques de Lyons et Guillaume Cotin. Mais tout doute cesse relativement à Jean Picard, qui fit, en 1604, un nouvel inventaire de la collection dont il avait la garde.

Cette dernière affirmation mérite d'être nuancée. Gourreau de la Proustière rappelle avoir exercé les fonctions de cellerier en plus de celles de bibliothécaire ${ }^{3}$ :

${ }^{2}$ A. Franklin, Les anciennes bibliothèques de Paris... Tome 1, Amsterdam, Gérard Th. Van Heusden, 1968, réimpression de l'édition de Paris 1867-1873 en 3 volumes, p. 150.

${ }^{3} \mathrm{Ph}$. Gourreau de la Proustière, Mémoires de Philippe Gourreau de la Proustière chanoine de Saint-Victor et curé de Villiers-le-Bel, texte établi et annoté par Béatrix de Buffévent. Paris, Fédération des sociétés historiques et archéologiques de Paris et de l'Île-de-France, 1990, p. 56. 
[...] Je fus cellerier à sa place [le P. Lhulier qui venait de mourir], charge fort contraire à l'estude, aussy en modérast-on les soins. Le P. Derieux, chambrier, ayant promis à la chambre qu'il me soulageroit, il ne manqua pas à sa parolle. Car si j'en avois le nom, il en portoit toute la peine, oultre qu'avec cet employ, j'estois encore bibliothécaire et je ne fus pas méry de joindre la bource aux livres, ayant plus de facilité d'en achepter qu'auparavant [...]

Pour sa part, Jean de Toulouse a été bibliothécaire alors qu'il était prieur. Mais d'une certaine façon, cela est assez compréhensible, compte-tenu du fait que dans toutes les communautés religieuses de quelque obédience qu'elles fussent, le supérieur a toujours été le directeur réel de la bibliothèque. On soulignera par ailleurs que la charge de bibliothécaire était l'une parmi d'autres dans une espèce de cursus honorum propre à la maison dans laquelle la plupart des religieux entraient vers l'âge de seize ou dix-sept ans. Il s'agissait d'une fonction intermédiaire que quelques-uns ont occupée après avoir été maître des novices, qui a pu être exercée plus ou moins longuement, et qui pour certains a préludé à l'accès à de plus hautes responsabilités dans la direction de la communauté. Mulot, par exemple, devint grand-prieur de Saint-Victor. Pour autant que nous le sachions, la fonction de bibliothécaire de cette abbaye n'a jamais requis de compétences particulières. Il n'y a rien là sans doute que de très ordinaire dans la plupart des communautés religieuses masculines de l'époque moderne. Pour d'autres, les fonctions de bibliothécaire ont été suivies de l'administration d'un prieuré-cure: Jean de Toulouse à Athis, Gourreau de la Prostière à Villiers-le-Bel...

\section{Comment connaitre les bibliothécaires de Saint-Victor?}

Avouons-le, cela est difficile, et beaucoup ne demeurent pour nous que des noms. Les dictionnaires biographiques, soit anciens soit encore inachevés, ne sont guère utiles, si ce n'est pour quelques personnalités un peu marquantes. Ainsi en estil par exemple de François-Valentin Mulot (1749-1804), davantage connu pour son implication dans la Révolution, comme député à la Législative, comme médiateur en Avignon en 1791, puis membre de la Commission des Monuments, et commissaire du directoire à Mayence, théophilanthrope ou ci-devant prêtre marié. Il l'est moins comme auteur de diverses brochures révolutionnaires, dont une Requête d'un vieil amateur de la Bibliothèque de Saint-Victor à l'évêque d'Autun, en vers, ou comme traducteur des Odes d'Anacréon, des Amours de Daphnis et Chloé de Longus (1782), des Fables de Lokman (1785), ou encore comme co-signataire avec Favart de Joseph ou Fin tragique de Mâme Angot, bagatelle morale mêlée de chants...

Un autre angle d'attaque consiste à interroger les écrits autobiographiques rédigés par quelques-unes de ces personnalités. Mulot, pour en terminer avec lui, a rédigé un Journal intime, pour la période 1777-1782, qui a été publié en 1902 par Maurice Tourneux ${ }^{4}$. Le manuscrit des Mémoires de Philippe Gourreau de la Proustière, né en 1661 et décédé en 1694, a été édité par Béatrix de Buffévent en

${ }^{4} \mathrm{M}$. Tourneux, Journal intime de l'abbé Mulot, bibliothécaire et grand-prieur de l'abbaye de Saint-Victor 1777-1782, publié par..., Mémoires de la Société de l'Histoire de Paris et de l'Île-de-France, Paris, Impr. De Daupeley-Gouverneur, 1902, p. 19-124. 
19905. Celui du Mémorial de Jean de Toulouse est désormais publié, par les soins de Jean-Baptiste Capit. Un premier volume, portant sur la période 1590-1637, a paru chez Brepols en 2001. Le second et dernier volume, couvrant les années 1638-1659, est sorti en $2008^{6}$. Pour sa part, Simon Gourdan, qui ne fut pas bibliothécaire, a rédigé des Vies et maximes saintes des hommes illustres qui ont fleuri dans l'abbaye de Saint-Victor, manuscrit encore inédit aujourd'hui conservé à la Bibliothèque Mazarine $^{7}$. Le premier constat qu'on doit faire est que les mémoires ou récits autobiographiques rédigés par des bibliothécaires de Saint-Victor, et auxquels nous venons de faire référence, sont peu diserts sur la vie de la bibliothèque et le travail qu'ils y ont accompli. D'une certaine manière, cela même est une information. Les responsabilités et tâches du bibliothécaire, sauf exception, relevaient d'un quotidien répétitif qui ne méritait pas de développement particulier. L'information qu'on retire de ces lectures est extrêmement ténue et fragmentaire. Jean de Toulouse ne dit rien de sa désignation comme bibliothécaire en 1636. Gourreau de la Proustière est presque plus disert sur sa bibliothèque personnelle, et sur celle du Vatican qu'il a visitée, que sur celle de Saint-Victor dont il a eu la charge. Ce constat n'a rien de bien surprenant. Il est valable pour l'étude de la bibliothèque de toute communauté religieuse quelle qu'elle soit. Il faudrait, pour en savoir davantage, passer beaucoup de temps à scruter les catalogues manuscrits conservés de la bibliothèque, avec leurs strates d'ajouts et de biffures. Il faudrait surtout dépouiller les textes régissant la vie de la communauté : règle, procès-verbaux et relevés de décision des chapitres et autres instances tant de la maison que de l'ordre dont elle relevait. Il faudrait enfin dépouiller tous les comptes et archives de gestion de la maison, type de documents dont on ne dispose pas forcément, afin d'y suivre les dépenses d'investissement et de fonctionnement faites pour la bibliothèque, par rapport aux revenus et aux autres dépenses de la maison : aménagement de locaux, achat de mobilier et de fournitures, acquisitions onéreuses, legs, dons et donations, frais de reliure... Seul un document de ce genre peut permettre de repérer des variations selon les mandats : périodes d'initiatives et périodes de léthargie... Il est bien évident qu'un tel travail est tributaire de l'état des sources conservées, et dépasse le cadre de cette modeste intervention.

\section{Quelles activités?}

\section{Administrer un lieu}

La localisation de la bibliothèque et son agencement a varié au cours de la période considérée. Les pères Jean de Toulouse et Gourreau de la Proustière rapportent un incendie survenu au dortoir le 5 janvier 1637. Le premier y perdit cinq volumes manuscrits des Annales de l'abbaye à la rédaction desquels il travaillait

${ }^{5}$ Cf. note 2.

${ }^{6} \mathrm{~J}$. de Toulouse, Le 'Mémorial' de Jean de Thoulouse, prieur-vicaire de Saint-Victor de Paris, tome 1, les années 1590-1637, édité par J.-B. Capit, Turnhout, Brepols, 2001-2008 (Bibliotheca victorina, 13, 1-2).

${ }^{7}$ S. Gourdan, Vies et maximes saintes des hommes illustres qui ont fleuri dans l'abbaye de Saint-Victor, Bibliothèque Mazarine, ms. 3348-3350. 
depuis 1625. Quant à Gourreau de la Proustière, il indique qu'un jeune homme s'était endormi sans souffler sa bougie, et il ajoutait ${ }^{8}$ :

c'est mal d'avoir fait loger les novices sous la bibliothèque, comme ce sont enfants sujets à dormir, il pouvait arriver un estrange inconvénient [...].

Il nous donne également une description rapide de cette ancienne bibliothèque :

[...] alors, ils estoient dans la bibliothèque, des manuscripts considérables par les estrangers. Les livres estoient couchez et enchaisnez sur de longs pipitres et une allée entre deux. Il y avoit quantité de petites fenestres entre chacune desquelles il y avoit un de ces tableaux avec des ornemens autour en camaïeux gris. Mais on a relevé ce bastiment du temps du priorat du P. Derieux en sorte qu'on a fait place à la bibliothèque que $\mathrm{M}$. Dubouchet Bournonville, conseiller en la grand chambre nous a donnée. Il n'y a plus de vestiges de l'ancienne bibliothèque qu'un tableau d'un religieux qui fait mine de monstrer à un ecclésiastique la bibliothèque, dans le novitiat, au-dessus de cette qui est maintenant, avec quelque grisaille.

En 1651, la bibliothèque fut inondée par une crue de la Seine. Les collections furent montées au second étage du bâtiment selon Franklin, au premier seulement selon Gilbert Ouy ${ }^{9}$. La salle pouvait contenir douze cents armoires. Elle était ornée de soixante-sept portraits des religieux de Saint-Victor les plus célèbres depuis la création de l'abbaye. Ces peintures avaient été faites à l'initiative de Gourreau de la Proustière $^{10}$ :

Je persuadai au P. Toulouse de faire paindre les hommes illustres de la maison, en dignité, science et piété, ce qu'il embrassat aisément, estant fort port\& pour l'antiquité dont il scavoit l'histoire. Un pintre nomé Toutain fit tous les portraits que l'on void encore au-dessus des tablettes de la bibliothèque, le $\mathrm{P}$. Thoulouse fournissant les noms, les dignités et la façon des habits.

Une double rangée de pupitres installée au milieu de la galerie permettait d'accueillir une cinquantaine de lecteurs. Près de l'entrée, une plaque de marbre rappelait le legs du Bouchet. Cette salle fut chauffée dès 1651. Une cellule contiguë servait de bureau au bibliothécaire. Un grand pavillon fut construit au bout de cette bibliothèque, pour y conserver à part les manuscrits. La césure dans la collection entre imprimés et manuscrits est donc relativement tardive. Nous n'avons aucune certitude que ce pavillon ait pu, aussi, servir de réserve pour les ouvrages les plus précieux, y compris les imprimés. Enfin, rien ne nous est dit des prohibiti, qui devaient être séparés du reste des livres, et sous clef.

${ }^{8} \mathrm{Ph}$. Gourreau de la Proustière, op. cit., p. 58.

${ }^{9}$ G. Ouy et al., Le catalogue de la bibliothèque de l'abbaye de Saint-Victor de Paris de Claude de Grandrue 1514, Paris, Éditions du CNRS, 1983.

${ }^{10} \mathrm{Ph}$. Gourreau de la Proustière, op. cit., p. 56-57. 
Nous avons vu combien déjà il était difficile de connaître les bibliothécaires de la maison. Nous savons par l'Almanach royal de $1709^{11}$ qu'ils étaient toujours choisis exclusivement parmi les religieux de l'abbaye. Nous savons également que le bibliothécaire était assisté d'un sous-bibliothécaire chargé de donner les livres au public des lecteurs. Ce soin fut, plus tard dans le siècle, confié à un simple "garçon». Quatre noms de sous-bibliothécaires nous sont connus: Guillaume Marcel décédé en 1708, Leperruquier en fonction en 1711, Bonamy attesté en 1722, Dadou son probable successeur. Quant aux garçons, seul le dernier, présent lors de l'apposition des scellés par les autorités révolutionnaires, est identifié. Il s'appelait Jean-Antoine Mathieu.

\section{Gérer une collection: Catalogues et récolements}

La tâche essentielle du bibliothécaire était de gérer les livres confiés à sa garde. Ce travail revêtait trois aspects principaux : rendre les ouvrages accessibles grâce à un catalogue précis, enrichir la collection par des acquisitions gracieuses ou onéreuses, organiser la communication des livres dans de bonnes conditions de sécurité. Le Catalogue des manuscrits de 1514, établi par Claude de Grandrue (Catalogus typographicus Bibliothecae manuscriptae Sti Victoris a Claudio de Grandivico canonico ejusdem abbatiae ordinatus anno 1514) servit aux récolements de ses continuateurs. Il fut revu par Jean Picard en 1604, puis par Jacques Bouët de la Noue en 1654, et par Le Tonnelier en 1664. Lors de l'arrivée du legs Du Bouchet en 1654, Bouët de la Noue effectua le premier récolement des manuscrits depuis Picard, un demi-siècle plus tôt ${ }^{12}$. La collection contenait alors environ 1500 manuscrits ${ }^{13}$ :

patient et méthodique, il s'efforce de situer chronologiquement chaque disparition. Il dépouille d'abord systématiquement les listes de manuscrits ou de cahiers perdus que Claude de Grandrue avait mis en appendice de son catalogue (Cecy manquait dès le temps que ce catalogue a esté fait en 1513), puis la série des 'revues' de ses prédécesseurs et son propre récolement de 1654.

Les disparitions notées par Bouët de la Noue, comme celles constatées dix ans plus tard par Le Tonnelier, posent le problème de la sécurité des collections et des vols, sur lequel nous reviendrons. Mais ce catalogue de 1514 fut prolongé, aux siècles suivants, par de nouveaux instruments de travail. En 1623, Étienne Reynard se lança dans la rédaction d'un nouveau catalogue alphabétique et méthodique en un volume in-quarto ${ }^{14}$. Jacques Bouët de la Noue prit sa relève. Eustache de Blémur, mort en 1691, avait entrepris un nouveau catalogue, lequel fut révisé, poursuivi et achevé en 1677 par Charles Le Tonnelier, qui lui avait succédé en $1664^{15}$. Ce

\footnotetext{
${ }^{11}$ Almanach royal pour l'an mil sept cens neuf..., éd. Laurent d'Houry, Paris, [1709], p. 218.

${ }^{12}$ G. Ouy et al., op. cit., p. XXXIII-XXXIV.

${ }^{13}$ Ch. Le Tonnelier, Annales ecclesiae Sancti Victoris Parisiensis..., p. 106.

${ }^{14} \mathrm{BnF}$, ms. fonds Saint-Victor 946.

${ }^{15}$ Ch. Le Tonnelier, Catalalogi Bibliothecae victorinae, tomus I, in tres partes distinctus. Prima pars : sacrorum Bibliorum textus; Secunda pars : elenchus auctorum qui in sacra Biblia universe vel singulatim data opera scripserunt, juxta eorumdem Bibliorum ordinem
} 
catalogue fut à nouveau revu en 1684 par Bouët de la Noue redevenu bibliothécaire. Dans son introduction à son catalogue de 1677, Charles Le Tonnelier avait souligné le caractère novateur de cet instrument de travail qu'il avait construit, puisque celuici était tout à la fois topographique, alphabétique et thématique ${ }^{16}$ :

Quelque soin qu'on apporte a ranger les livres d'une biblioteque nombreuse, les différentes matières des auteurs, jointes aux différentes grandeurs des livres, ne permettent pas d'y apporter un ordre parfaict; si bien que, malgré que l'on en ayt, on est obligé de mesler les matières les unes avec les autres sans distinction, et d'avoir recours aux catalogues, tant pour faciliter la mémoire des bibliothécaires, et donner par ce moyen une cognoissance facile aux estrangers des trésors qui y sont renfermés que pour rectifier ce qui y est défectueux. Il en fault trois :

Le premier sera selon l'ordre des tablettes,

Le second doit suivre la lettre alphabétique,

Et le troisiesme selon les différentes matières.

C'est l'ordre que nous avons suivy dans la Biblioteque de Saint-Victor.

Monsieur de Blemur, mon predecesseur, dont les hautes qualités luy ont acquis à justes tiltres les premieres dignités de cette abbaye, et est a present prieur de Saint Guenault de Corbeil, membre dependant de l'abbaye de Saint-Victor, employé dans toutes les grandes affaires du diocese, a commencé le premier, qui est selon l'ordre des tablettes, et nous, à son imitation, avons continué ce mesme volume, et enfin terminé. Plus nous avons faict le second, par ordre alphabetique, lequel contient trois volumes in-folio escrits de ma main. Ils ne sont pas dans la derniere exactitude, quoique j'y aye apporté beaucoup de soin et d'application. La difficulté qu'il y a de conserver plusieurs petits papiers entassés les uns sur les autres pour former cest ordre alphabetique, particulièrement pendant la chaleur de l'esté, qu'on est obligé de se donner de l'air; le mesme zéphir qui vous resjouit de sa fraischeur a trouiblé l'ordre qui avoit esté mis dans ces papiers pour executer le dessein qu'on avoit projetté, disgrace qui m'est arrivé trois fois, et qui est cause des fautes qui s'y rencontrent. [...] Ceux [les livres] qui sont anonymes, vous les trouverés sous la lettre de la matiere dont-ils traitent: comme s'ils traitent de la penitence, vous trouverés l'auteur sous la lettre $P[\ldots]$ Chaque volume est costé à deux lettres et un chiffre. La premiere est la lettre qui regne tout le long de l'armoire; la seconde est celle de la planche, et le chiffre matrque le rang que tient ledit auteur sur sa tablette [...] Pour ce qui est du troisiesme, qui est selon les matières, il contiendra plusieurs tomes $[\ldots]$.

Le rôle de tout bibliothécaire, partout, a toujours été de développer et d'enrichir les collections confiées à sa garde. Seul un dépouillement précis des archives financières de l'abbaye permettrait de mettre en lumière les politiques successives d'acquisitions, et le coût des trains de reliures qui les accompagnaient,

dispositus; Tertia pars: index chronologicus secundum ordinem saeculorum distributus, sanctorum patrum aetates et scripta indicans; ad calcem auctores imperfecti temporum injuria, opera et studio Caroli Le Tonnellier, bibliothecarii., 1677, Bibliothèque Mazarine, ms 4188 (anciennement 3265).

${ }^{16}$ A. Franklin, op. cit., p. 182-183. 
puisque la reliure était alors la seule méthode de conservation préventive que l'on connaissait. Les enrichissements de la bibliothèque se sont faits de différentes manières. Il y eut une part, sans doute importante, d'acquisitions à titre onéreux. C'est en effet par ce biais qu'un bibliothécaire pouvait exercer ses choix sans être tenu par autre chose que la politique documentaire qu'il avait décidée. Nous savons ainsi par Jean de Toulouse que Philippe Gourreau de la Proustière acheta dix-huit manuscrits ${ }^{17}$. Ce dernier écrit pour sa part $^{18}$ :

[...] j'achepté beaucoup de livres tant imprimez que manuscrits ou que j'échangé contre d'aultres que nous avions doubles. Comme j'achepté Saint Thomas, touttes ses œuvres d'impression d'Envers [Opera omnia, Anvers, 1612, 18 volumes infolio] ; touttes celles de Saint Cyrille [Paris, J. Aubert, 7 volumes in-1, les Conciles généraux et aultres [Conciliorum omnium generalium et provincialium collection regia, Paris, 1644, 37 volumes in-folio].

Nous avons là une illustration, parmi d'autres, de la constitution par la quasitotalité des communautés religieuses de volumineux instruments de travail, fruits de la politique éditoriale de la Réforme catholique, et qui devaient servir des générations de religieux des $\mathrm{XVII}^{\mathrm{e}}$ et $\mathrm{XVIII}^{\mathrm{e}}$ siècles. Pourtant, la conjoncture économique limitait parfois ces acquisitions. Nous savons qu'en 1684, les rentes furent tout juste suffisantes pour payer le personnel. Nous savons aussi que depuis la réduction sur les rentes, intervenue en 1720, la bibliothèque était réduite à un revenu de 1503 livres 9 sols 6 deniers, provenant de différents legs qui lui avaient été faits. Dans un tel contexte, les libéralités extérieures étaient bienvenues. Elles provenaient pour partie de legs de leur bibliothèque personnelle effectués par des religieux de l'abbaye. Tel fut par exemple le cas de Jean Picard qui mourut de la maladie de la pierre le 15 juin 1615, abandonnant à Saint-Victor une partie des livres lui appartenant. D'autres libéralités émanaient de l'extérieur. Gourreau de la Proustiere mentionne ${ }^{19}$ un présent reçu vers 1666 de quatre vingt onze volumes in-folio sortis des presses de l'imprimerie royale, reliés « en maroquin du Levant».

La libéralité la plus somptueuse dont bénéficia Saint-Victor fut certainement la donation faite par Henri du Bouchet de Bournonville en $1652^{20}$ :

[...] desirant que le P. Eustache de Blemur, qu'il tesmoigne estre son amy, et en effect promoteur de ce bienfaict, prenne le soing de ceste bibliotheque tout le temps qu'il sera residant en ceste Maison, et en prenne la direction, nonobstant les reffus humbles qu'il luy avoit faict plusieurs fois de cette grace.

Les livres arrivèrent à l'abbaye en 1654. Enfin, les bibliothécaires de SaintVictor surent, en quelques occasions, récupérer des livres distraits plus ou moins anciennement de leur bibliothèque. Ainsi en, fut-il, par exemple, de six beaux manuscrits que le P. Jean de Toulouse, peu après sa prise de fonction en qualité de

\footnotetext{
${ }^{17}$ Ibid., p. 160.

${ }^{18} \mathrm{Ph}$. Gourreau de la Proustière, op. cit., p. 57.

${ }^{19}$ Idem, p. 417-418.

${ }^{20}$ A. Franklin, op. cit., p. 154.
} 
bibliothécaire en 1636, avait donnés au noviciat des Jésuites, qui venait d'ouvrir. Saint-Victor les racheta pour 49 livres 8 sols lors de la suppression des Jésuites.

$\mathrm{Au}$ milieu du XVIII ${ }^{\mathrm{e}}$ siècle, la bibliothèque aurait abrité environ 35000 imprimés et 3000 manuscrits. Elle était ouverte De 8 à 10 heures, et de 14 à 16 heures (17 heures en été) les lundis, mercredis et samedis. Elle était fermée deux mois durant l'été, du 15 août à la saint Luc (18 octobre $)^{21}$. Le bibliothécaire pouvait, à tout moment, retirer un ouvrage de la salle de lecture, soit pour l'emporter dans son bureau, la cella bibliothecarii, soit pour le protéger en raison ou de sa fragilité ou de la convoitise de lecteurs indélicats. Une note de la main de Picard en marge de la notice du manuscrit CCC1 du catalogue indique : relegatus est consulto in cellam bibliothecarii. Cette pratique a sans doute existé de tout temps.

Comme toutes les grandes bibliothèques de Paris, celle de Saint-Victor était un lieu qu'il fallait visiter. Aux lecteurs habituels, chanoines de l'abbaye et gens du dehors, s'ajoutaient régulièrement les personnalités françaises ou étrangères de passage. Il était alors du devoir du bibliothécaire de se tenir à la disposition de ces hôtes. Gourreau de la Proustière nous le rappelle en passant: "Je monstrois la bibliothèque aux estrangers qui avoient grande curiosité pour cela $»^{22}$. Quant au $\mathrm{P}$. Toulouse, il relevait à la date du 22 avril 1636 :

[...] et m'en revins le lendemain du grand matin pour me tenir à la maison pour estre prest à monstrer la maison et la bibliothèque au duc Bernard de Saxe-Weimar, qui disnoît ce jour là au Jardin royal, en cas qu'il eust eu désir de venir veoir cette bibliothèque, mais la carousse alemande détourna ce projet. ${ }^{23}$

Dans une bibliothèque accueillant du public, où nombre de livres étaient à portée de main du lecteur ou du visiteur, l'une des hantises du bibliothécaire demeurait le vol. Or nous savons que de nombreux larcins ont été commis dans la seconde moitié du $\mathrm{XVI}^{\mathrm{e}}$ siècle, puis encore sous l'administration du P. Jean Picard mort en 1615. Naïf et distrait, ce dernier était aux dires de Gourreau de la Proustière

un peu trop facile à se laisser dérober des manuscrits, qu'on luy coupoit tandis qu'il entretenait d'autres. Un M. Petaut conseiller [...] et d'autres ont fait ce tort au public [..] Le fils Petaut a vendu ses manuscrits à la reine de Suède : la restitution en sera difficile, oultre qu'ils n'en font pas grand cas de conscience. ${ }^{24}$

Un autre voleur d'envergure finit par être confondu dans les années 1640 : l'érudit allemand Heinrich Lindenbrog. Selon le témoignage du théologien protestant Paul Colomiès ${ }^{25}$ :

[...] on alla le prendre un matin en bonnet de nuit et en pantouffles et qu'on le mena ainsi en prison, d'où il sortit quelques jours après par le crédit de M. Du Puy.

\footnotetext{
${ }^{21}$ G. Ouy et al., op. cit., p. XV.

${ }^{22} \mathrm{Ph}$. Gourreau de la Proustière, op. cit., p. 57.

${ }^{23}$ J. de Toulouse, op.cit., p. 534.

${ }^{24}$ G. Ouy et al., op. cit., p. XVII.

${ }^{25}$ G. Ouy et al., op. cit., p. VIII.
} 
Ces différents épisodes malheureux expliquent certaines réticences à accueillir du public et à communiquer des ouvrages qui se firent jour au XVIII ${ }^{\mathrm{e}}$ siècle. Il est enfin une dernière tâche qui incombait au bibliothécaire, celle d'assister les religieux de la maison à publier leurs œuvres, en les mettant en relation avec des imprimeurs-libraires compétents, ou en les assistant dans leurs démarches en vue d'obtenir un privilège du roi. Le P. Jean de Toulouse nous en rapporte un exemple qui plaide pour d'autres cas non relatés par nos sources ${ }^{26}$ :

En 1608, le P. Jean Picard, bibliothecaire et nostre maistre de latin, fist mettre soubz la presse le livre de notre P. souprieur Garnerus intitulé Gregorianum ${ }^{27}$, parce qu'en iceluy il faict un recueil des plus belles moralitez et doctes recherches de tous les livres de Sainct Gregoire, pape et docteur de l'Église.

Après avoir rappelé certaines des tâches les plus essentielles qui incombèrent aux bibliothécaires successifs de Saint-Victor, il apparaît légitime de s'interroger sur leurs conceptions et pratiques bibliothéconomiques.

\section{Quelle bibliothéconomie?}

Répondre à cette question est très délicat. Il faudrait pour ce faire disposer des archives comptables de la maison, et scruter les relevés de conclusions des chapitres de l'ordre et de l'abbaye. Il nous faut nous souvenir également que le $\mathrm{XVII}^{\mathrm{e}}$ siècle fut une époque durant laquelle s'élaborèrent et furent théorisées des conceptions et des pratiques de gestion des bibliothèques qui n'ont certainement pas manqué d'être connues, et peut-être adoptées, par les bibliothécaires successifs de la maison.

\section{Un savoir professionnel en cours d'élaboration}

On rappellera à cet égard un certain nombre d'ouvrages majeurs parus à cette époque. L'un des premiers fut sans conteste le De bibliothecis syntagma paru en 1602 sous la signature de Juste Lipse ${ }^{28}$. Dans ce petit in-quarto de 34 pages, l'auteur, après avoir brossé à grands traits une histoire des bibliothèques depuis l'antiquité, entreprenait de convaincre le duc de Croÿ de bâtir une bibliothèque et de l'ouvrir au public des beaux esprits pour faire «éclater son nom ». Ce premier manifeste pour l'ouverture des collections privées au public a trouvé un continuateur en la personne de Gabriel Naudé, alors bibliothécaire du président de Mesmes, qui publia en 1623 son Advis pour dresser une bibliothèque, lequel connut une seconde édition en

\footnotetext{
${ }^{26}$ J. de Toulouse, op.cit., p. 156-157.

${ }^{27}$ Garnerus, Gregorianum... hoc est, allegoricae omnium pene rerum in Bibliis contentarum explanationes promptae ex universis D. Gregorii papae scriptis..., Parisiis, Carolus Seuestre, $1608,8^{\circ}$.

${ }^{28}$ J. Lipse, Justi Lipsi de bibliothecis syntagma, Antverpiae, ex officina Pllantiniana, apud Ioanem Moretum, 1602, $4^{\circ}$.
} 
$1644^{29}$. Ces deux textes s'adressaient à de grands personnages, et non à des communautés religieuses. Pourtant, l'abbaye de Saint-Victor en a peut-être retiré un certain nombre d'enseignements puisqu'elle fut l'une des rares bibliothèques parisiennes à accueillir des lecteurs extérieurs à la communauté, et à laisser à leur disposition de nombreux ouvrages et usuels alignés dans les armoires garnissant les murs, à portée de main, dans une espèce de «libre accès » avant la lettre. En accueillant ces lecteurs, elle se conformait aux vœux exprimés dans son testament par l'un de ses principaux mécènes, Henri du Bouchet de Bournonville ${ }^{30}$ :

[...] Item, ayant avec beaucoup de peine et de soin travaillé depuis long-temps à faire une bibliothèque, et composé icelle des meilleurs livres qu'il a pû recouvrer, desirant qu'elle soit exactement conservée et entretenuë pour le bien du public à perpétuité, il a estimé ne pouvoir faire un meilleur choix que de la mettre, comme un depost, entre les mains de Messieurs les Chanoines reguliers de l'abbaye dudit S. Victor-lez-Paris, auxquels il fait don et legs de sa dite bibliothèque [...] aux conditions suivantes : qui sont que les gens d'étude auront la liberté d'aller étudier en la biblioteque de ladite abbaye, où lesdits livres seront transportez, mis, et tenus au meilleur ordre qu'il se pourra, trois jours de la semaine, trois heures le matin et quatre heures l'apresdiné, lesquels jours seront le lundy, mercredy, et samedy; et, s'il arrive qu'il soit fête lesdits jours, il sera fait remise aux jours suivans, dont le bibliotequaire donnera avertissement; et à cette fin lesdits religieux seront tenus de faire que l'un d'eux se trouve ausdits jours et heures en ladite biblioteque, pour avoir le soin de bailler et de remettre les livres, après que les étudians en auront fait. [...]

Si l'ouvrage de Gabriel Naudé inspira peut-être le choix du généreux donateur, puis de ceux qui l'imitèrent par la suite, il est un autre ouvrage qui a pu influencer les bibliothécaires de Saint-Victor, celui du jésuite franc-comtois Claude Clément, en poste à Madrid, qui publiait à Lyon en 1635 son Musei sive bibliotheca.... libri quatuor ${ }^{31}$. Ce gros volume insistait beaucoup sur la monumentalité qui devait être celle d'une bibliothèque, et sur l'élaboration d'un décor allégorique, dont toute la symbolique devait pénétrer le visiteur et le lecteur. Mais ce livre avait un autre intérêt. Alors que Gabriel Naudé restait dans des considérations générales sur les conditions de rangement et de conservation des ouvrages, Clément était sans doute le premier auteur à donner des informations plus techniques, en particulier sur la prévention contre les moisissures. Il est vrai que cette information est à chercher derrière tout un fatras de considérations mythologiques, sans doute fort communes en son temps mais déroutantes aujourd'hui. Un autre ouvrage, postérieur d'une génération, ramasse en une quinzaine de pages le premier traité de bibliothéconomie technique du temps : les Règles communes et particulières de la congrégation de Saint-Maur. Publié une

${ }^{29}$ G. Naudé, Advis pour dresser une bibliotheque presenté à Monseigneur le Président de Mesme. Par G. Naudé..., Paris, chez François Targa, 1627, $8^{\circ}$, seconde édition, Paris, chez Rolet le Duc, $1644,8^{\circ}$.

${ }^{30}$ A. Franklin, op. cit., p. 181.

${ }^{31} \mathrm{Cl}$. Clément, Musei sive bibliotheca tam privatae quam publicae extructio, instructio, cura, usus, libri IV..., Lugduni, sumptibus Jacobi Prost, 1635, $4^{\circ}$. 
première fois en 1663 , l'ouvrage fut réédité sans changement en $1686^{32}$. Il se compose d'une suite de chapitres qui présentent ce que devaient faire et ne pas faire les religieux qui exerçaient des fonctions au service de la communauté : portier, jardinier, cuisinier, bibliothécaire... Tous ces textes sont anonymes, mais il est permis de penser que Dom Tarisse ne fut sans doute pas totalement étranger au chapitre intitulé «Règles du bibliothécaire», qui couvre les pages 78-93 de la première édition de l'ouvrage. Ce petit texte est organisé en six parties techniques :

de la disposition de la bibliothèque

de l'ordre des livres

des catalogues de livres

de la distribution des livres

de l'achat et du choix des livres

du soin et de l'entretien de la bibliothèque

De fait, il présente un état des pratiques bibliothéconomiques, issues de l'expérience de générations successives de bibliothécaires, en cours à l'époque considérée. Sa portée s'étend bien au-delà de la Congrégation de Saint-Maur qui en a assuré la rédaction et la publication ${ }^{33}$. Les principes qu'il énonce ne contredisent en rien le règlement de la bibliothèque, d'après la Règle générale de Saint-Victor, que Franklin traduit du latin dans son ouvrage :

Le bibliothécaire a sous sa garde tous les livres de l'église (c'est à dire de la maison ou de l'abbaye, ou, si l'on veut, de la communauté). Il doit en posséder une liste nominale, et au moins deux ou trois fois par an les feuilleter, en faire l'inventaire, et examiner soigneusement s'il ne s'y trouve ni vers qui les ronge ni rien qui les salisse. La bibliothèque doit être intérieurement garnie de boiseries, de peur que l'humidité des murailles ne se communique aux manuscrits et ne les détériore. Les pupitres ne doivent être ni trop rapprochés ni trop éloignés les uns des autres ; et les livres qui y sont placés ne seront ni trop serrés, ni trop éloignés les uns des autres ; et les livres qui y sont placés ne seront ni trop serrés, ce qui pourrait nuire à leur conservation, ny trop écartés, ce qui pourrait y amener de la confusion et entraver les recherches. Le bibliothécaire tiendra à la disposition de tous les livres qui sont nécessaires pour le travail de chaque jour, de même que ceux qu'il juge plus spécialement applicables à l'instruction et à l'édification des religieux : tels sont les Bibles, les Vies des Pères et les principaux commentateurs sacrés. Quant aux autres ouvrages, qui sont d'un usage moins général, ils resteront toujours dans la bibliothèque, et le bibliothécaire ne s'en déssaisira que sur la demande formelle d'un

\footnotetext{
${ }^{32}$ Règles communes et particulières de la Congrégation de Saint-Maur, Paris, [s.n.], 1663, $8^{\circ}$. Règles communes et particulières de la Congrégation de Saint-Maur. Nouvelle édition corrigée sur les constitutions déclarations et réglemens de Chapitres Généraux, [s.l., s.n.], $1686,8^{\circ}$

${ }^{33}$ D. Varry, «Contribution à une histoire des savoirs professionnels des bibliothécaires : les 'règles' du bibliothécaire mauriste », Facettes. Réflexions multiples sur l'information. Textes réunis et présentés par Chantal Dentzer-Tatin, Lyon, Association des Documentalistes et Bibliothécaires Spécialisés (ADBS) Rhône-Alpes, 1994, p. 177-185.
} 
religieux ; dans ce cas, il prendra aussitôt note des livres prêtés, afin qu'il lui soit toujours facile de savoir quels ouvrages possède chaque religieux. Ceux qui empruntent des livres doivent les conserver avec beaucoup de soin, ne pas les confier à d'autres personnes, et, quand ils n'en ont plus besoin, les remettre au bibliothécaire. ${ }^{34}$

Au contraire, il vient le conforter par des consignes précises. Naudé, Clément, Dom Tarisse... nous rappellent qu'on ne s'improvise pas bibliothécaire, mais qu'on le devient. Or nous devons bien avouer tout ignorer de la façon dont, à Saint-Victor, le témoin se passait d'un bibliothécaire à l'autre. Notre documentation ne nous permet pas d'assurer que le nouveau bibliothécaire avait préalablement exercé les fonctions de sous-bibliothécaire auprès de son prédécesseur. Seul le cas du P. Jacques Bouet de la Noue le laisse supposer puisqu'il semble avoir été «assistant» de 1641 à 1648 , puis «titulaire» de 1648 à 1651 , et une nouvelle fois après 1684. Comme en beaucoup d'autres endroits, le métier devait s'apprendre sur le tas, et de bouche à oreille. Cet état de choses, comme la durée d'exercice assez courte, ne devait guère être favorable au développement d'une bibliothèque. En tout état de cause, nos mémorialistes sont muets sur cet aspect de leurs carrières respectives.

\section{communauté \\ Les rapports entre le bibliothécaire et le supérieur de la}

S'il est un autre sujet très peu abordé par les textes interrogés, c'est bien celui-ci. Or, dans une communauté religieuse de l'époque moderne, quel que fût l'ordre auquel elle se rattachait, le bibliothécaire n'était jamais qu'un « technicien d'exécution». Rappelons-nous qu'à Saint-Victor, les fonctions de bibliothécaire étaient d'un niveau intermédiaire dans la hiérarchie des charges. Toutes les décisions relevaient du supérieur: l'agencement des locaux, le choix des acquisitions, les autorisations d'admettre ou non des lecteurs extérieurs, les autorisations de communication et de prêt... Le véritable directeur de la bibliothèque était toujours le supérieur de la maison, et il avait toujours un contrôle théorique sur les lectures de ses religieux. La latitude du bibliothécaire était donc réduite. Quand, comme dans le cas de Jean de Toulouse, il était aussi sous-prieur, les choses devaient être plus faciles. Mais certains témoignages de Gourreau de la Proustière ${ }^{35}$ laissent supposer, à propos du P. Picard, des difficultés qui durent se poser pour d'autres :

Le bon homme Picart est mort usé d'estudes et accablé de cloistre. On est malheureux de rencontrer quelque foys des supérieurs jaloux et ennemys des estudes et contraires aux estudians. Ils n'en veulent point de plus éclairez qu'eux. Ils travaillent et assujetissent ceux qui ont disposition aux estudes et qui sont en estat de paroistre. Ils les obscurcissent et surchargent, les faisant avorter. Et la rosée estant preste de former une bonne perle dans la coquille, ils n'en font qu'une brute et un avorton par leurs bruits. Il fault qu'ils estudient en secret ou s'ils quittent l'estude par ces contradictions, ils se débauchent et voylà le fruit et la gloire de ces

\footnotetext{
${ }^{34}$ A. Franklin, op. cit., p. 150-151.

${ }^{35} \mathrm{Ph}$. Gourreau de la Proustière, op. cit., p. 128.
} 
supérieurs ombrageux et superbes. [...] Et comme les scavans sont d'ordinaire plus clairvoyans, les supérieurs apréhendent qu'ils ne découvrent leurs défaults et qu'ils ne soient autant de censeurs de leur conduitte. Le pauvre Père Picard a un peu essuyé, sur ses vieux jours, de ces caprices prioralles.

Il est d'ailleurs possible que les clauses du legs Bouchet de Bournonville, et des suivants, avec leurs conditions d'ouverture au public aient créé des difficultés avec la vie et les exigences de la communauté... et aient tiraillé le bibliothécaire entre des exigences contradictoires. C'est peut-être ainsi qu'il faut entendre le propos prêté par Montesquieu au supérieur de Saint-Victor dans la conversation qu'il aurait eu avec le visiteur persan Rica ${ }^{36}$ :

J'ai mon bibliothécaire qui vous donnera satisfaction : car il s'occupe nuit et jour à déchiffrer tout ce que vous voyez là ; c'est un homme qui n'est bon à rien, et qui nous est très à charge, parce qu'il ne travaille point pour le couvent.

Cette rapide présentation ne répond sans doute que bien imparfaitement à la question posée : qui étaient les bibliothécaires de Saint-Victor aux XVII ${ }^{\mathrm{e}}$ et $\mathrm{XVIII}^{\mathrm{e}}$ siècles? Elle conforte des informations que nous connaissions déjà pour d'autres maisons et d'autres ordres. Elle nous permet de comprendre combien cette bibliothèque était conforme au modèle de son temps par la réorganisation de locaux fonctionnels, la constitution d'instruments de travail à partir de la production de la Contre-Réforme, l'ouverture au public... Elle nous suggère même que Saint-Victor était, par certains aspects, en pointe sur les pratiques du temps. Ainsi en est-il de cet embryon de «libre accès» qui permettait au lecteur de tirer certains usuels des tablettes sans être obligé de les demander préalablement au bibliothécaire. La rançon de ces pratiques plus libérales qu'ailleurs est sans doute les vols et dégradations signalés. Beaucoup de bibliothécaires ne demeurent pour nous que des noms. Leur court temps d'exercice ne leur a sans doute pas permis d'avoir de politiques ambitieuses, à la différence de certains grands noms du XVII ${ }^{\mathrm{e}}$ siècle. Dans tous les cas, le poids du supérieur, véritable bibliothécaire en chef, et dont nous ne savons rien, dut être considérable, facilitant ou entravant les initiatives... Après tout, pour beaucoup, la charge de bibliothécaire n'a été qu'un épisode, ni plus ni moins marquant que d'autres, de leur vie à l'abbaye. Pouvons-nous espérer en découvrir davantage? Il faut alors retourner aux archives comptables et au catalogue des imprimés. Le véritable travail est encore à mener...

\section{Dominique Varry Université de Lyon, Enssib, Centre Gabriel Naudé, EPHE IV ${ }^{\mathrm{e}}$ section}

\footnotetext{
${ }^{36} \mathrm{Ch}$. de Montesquieu. Euvres complètes I, texte présenté et annoté par Roger Caillois, Paris, Gallimard, 1949, collection de la Pléiade. Lettres persanes, lettre CXXXIII, p. 331.
} 